\title{
Approach to patient with cough by American and Chinese respiratory specialist
}

\author{
Xiuxiu Liu ${ }^{1}$, Deniz Kesebir ${ }^{2}$, and Mikhail Kazachkov² \\ ${ }^{1}$ Shanghai Longhua Hospital Affiliated to Shanghai University of Traditional Chinese \\ Medicine \\ ${ }^{2}$ New York University School of Medicine
}

May 13, 2020

\begin{abstract}
Cough is a common pediatric complaint. Acute cough is often considered to be self-limiting and not requiring particular management. However, pediatric patients and their parents often seek remedy for acute cough. Traditional Chinese Medicine (TCM) Specialists have been treating pediatric cough for millenniums. Here we present a case of pediatric cough and approach to it by Western and Chinese Respiratory Specialist. We conclude that TCM may provide important and useful insight on treatment of pediatric respiratory diseases.
\end{abstract}

\section{Background}

Cough is one of the most common complaints in pediatrics. ${ }^{12}{ }^{2}$ Approach to cough by Western Pediatricians is defined by guidelines, which are based on published studies and opinions of experts in the field, ${ }^{4}$ however, controversy still exists. The pediatric cough is divided into acute and chronic. Chronic cough is typically diagnosed after at least 4 weeks of coughing and is often associated with significant respiratory morbidity. ${ }^{6}$ Acute cough is mostly attributed to viral respiratory illnesses and, at least according to academic dogma is considered "self-limiting" and is not requiring particular management. In fact, usage of cough medicine is somewhat discouraged, particularly in smaller children, who were shown to have more side effects from it (REF). ${ }^{7}$ Nevertheless, acute cough often becomes difficult for the patients and worrisome for the family, and it is only natural for the parents and, in many instances, for the pediatricians to seek remedy for this cough.

Traditional Chinese Medicine (TCM) specialists have been treating pediatric cough for millenniums. Its concepts of diagnosis and treatment have stood the test of time. Practitioners of TCM believe that the immature respiratory and immune system of children make them more susceptible to infections and environmental irritants. They also believe in strong connection between respiratory symptoms and dysfunction with the other parts of the body, such as kidneys and spleen. According to the TCM theory, Qi, which is a person's vital energy, acts as protective mechanisms against disease. Children have a relatively weaker Qi compared to adults and thus will become ill more frequently and will recover more slowly. Their lungs are delicate, and their defense systems are easily breached by exogenous "pathogenic evils". In TCM, "six pathogens" or "six evils" (wind, cold, heat, dryness, dampness, summer heat) are thought to take advantage of extreme weather changes and the body's transient weakness. According to TCM theory, the lungs are not only responsible for respiration, but also dominate the Qi of the whole body. Qi is particularly susceptible to pathogenic wind, leading to failure of the lungs Qi to disperse and descend, which may manifest with coughing. ${ }^{8}$ Although, wind evil is the most common pathogen to invade the lungs, wind can co-infect with other evils such as cold and heat to cause illness in the lung. ${ }^{9} 10$ 
If the cough is due to an exogenous evil that is not properly treated, lung-Qi may be injured and cough may become protracted and difficult to treat.

TCM recognizes six types of pediatric coughs: wind-heat, heat phlegm, wind-cold, damp phlegm, yindeficiency and qi-deficiency, each of which presents differently. TCM physicians look at the main characteristics of the cough - sound, frequency and sputum. They also perform careful physical examination, which include assessment of color of the tongue and pulse. Treatment is highly specific and depends on physician's conclusion on the type of the cough. For example, "heat phlegm cough", which is characterized by production of sticky phlegm, is treated with cooling, moistening herbs and acupuncture points that clear heat from the lungs. On the other hand, "wind-cold cough", a cough due to cold, which is accompanied by chills and copious mucus is treated with warming, drying herbs and acupuncture.

Similarly to western medicine, TCM seeks "to treat the child, no the cough". Treatments are individualized and are based on condition, age, weight and other factors, and different children with the same condition may be treated differently. The common thread that runs through all treatment is the elimination of imbalances in the body and restoration of Qi flow.

Apparently, there is substantial difference in the approach to cough between American and Traditional Chinese Medicine Respiratory Specialists. In order to understand the differences and similarities in the approach to patient with respiratory symptoms, we present a case of pediatric cough managed by American Respiratory Specialist (ARS) and Chinese Respiratory Specialist (CRS) with expertise in TCM.

\section{Methods}

This case study was performed as a Quality Improvement Project, and families volunteered and consented to participate. Each patient was approached consecutively by ARS and CRS. The history was taken, and physical examination was performed. It included thorough assessment of tongue and pulse by CRS.

\section{Clinical case}

\section{ARS}

Three year old male with history of asthma presents to the clinic with 14 days of cough.

He had low-grade fever and "congestion" for three days in the beginning of illness.

His cough was strong, forceful and wet, with production of white-clear sputum. The cough bothered him predominantly at night at bedtime. The cough attacks, lasted for 20-30 seconds, there was post-tussive vomiting 2-3 times a day. He had no shortness of breath during the day while playing and no wheezing.

His medical history was significant for asthma diagnosed by his pediatrician. He was prescribed inhaled Fluticasone (Flovent) $110 \mathrm{mcg} 2$ puffs BID one month ago, but the family admits to irregular usage of this treatment. He had no allergies. His mother also has asthma.

His vital signs are normal for his age; $\mathrm{SpO} 2$ is $98 \%$ on room air

His physical examination, revealed normal non-toxic appearing child, with a wet and strong cough. He had clear nasal secretions, hyperemia of pharyngeal mucosa. Inspection and palpation of the chest were unremarkable; percussion of the chest reveals resonant sound over the lungs. Auscultation of the chest was significant for soft rattling sounds; no wheezing and no crackles. He had no clubbing.

Impression: The $w$ et cough is likely related to a viral illness, the lingering cough with history of asthma and family history of asthma, is due to asthma; therefore, this is a likely presentation of subacute viral induced asthma.

Plan: Flovent $110 \mathrm{mcg} 2 \mathrm{p}$ bid with valve holding chamber (VHC) and mask to treat the airway inflammation due to asthma. Albuterol 2-4 puffs every 4 hours with VHC and mask as needed for wheezing/cough for possible airway reactivity due to asthma. 
ARS Summary : Child with wet cough related to viral illness. It is hard to rule out exacerbation of asthma. Asthma medications have to be resumed. Follow up needs to be provided in 2 weeks. If wet cough fails to improve, course of antibiotics for presumed protracted bacterial bronchitis shall be considered.

\section{CRS}

\section{TCM History Taking}

Chills and Fever: present at onset of illness

Abnormal sweating: none

Head, body, chest and abdomen complaints: none

Changes in appetite and abnormal taste in the mouth : none, but the mother reports "bad eating habit and eats too much desserts"

Sleep disorders and mental impairments : none

Constipation, diarrhea and urination complaints : none

Physical Exam

Vital Signs: Temperature $37.1^{\circ} \mathrm{C}\left(98.7{ }^{\circ} \mathrm{F}\right), \mathrm{SpO} 298 \%$

Nasal Congestion: stuffy and runny nose

Abnormal chest sounds: coarse breath sounds to auscultation bilaterally

Pulse-taking and palpation : floating, tight

Tongue inspection: white and thin tongue coating

Abdominal exam: soft, non-tender, non-distended

TCM diagnosis: Cold Cough. Syndrome differentiation: wind-cold exogenous pathogenic attack lung

Plan:

Prescription: Jin Fei Cao San (Inula Decoction)

Auricular Acupuncture: Twice a week, press the auricular points with finger down on the mustard seeds three times a day for around one minute each time. Ear points: Lung, Trachea, Shen-Men

Diet: Avoid excessive desserts

\section{CRS Summary}

This case was marked by cough, clear thin sputum, stuffy and runny nose, white and thin tongue coating due to failure of the lung Qi (vital energy) to ascend and descend because of external disturbance of the lung by wind-cold exogenous pathogenic factors. It is classified into the TCM disease category of "exogenous cough". Jin Fei Cao San (JFCS) is a formula quite effective for this type of cough (table 1).

Auricular acupuncture is a treatment system based on normalizing body's dysfunction through stimulation of points on the ear. According to TCM, the ear has a close relationship with the viscera and meridians. There are corresponding reaction areas (auricular points) in the auricle. ${ }^{11}$ This treatment aims restoring the flow of Qi by resolving any blockages or disruption. ${ }^{1213}$

Also noteworthy is the child's poor eating habits and his preference for desserts. TCM postulates "children's digestion is insufficient." This statement particularly applies to children younger than six years of age. The immature digestion combined with an improper diet accounts for eczema, cough, allergies and asthma. It is believed that excess of sweet food increases burden on spleen, which is considered a source of phlegm. ${ }^{14}$ In 
addition to treatment, it is important for this child to improve his diet. He should opt for light and digestible food, and refrain from taking oily food to reduce the burden on the spleen.

\section{Discussion}

We presented a patient with acute cough, which was evaluated consecutively by Western and Chinese Respiratory Specialists, who came to their conclusions on diagnosis and treatment. There are significant differences in their approach to the case. ARS attributed cough mostly to viral illness and suggested that it also could be consistent with asthma flare. As it was expected, cough medicine was not recommended and the emphasis was put on proper asthma management. Consideration was given to antibiotic treatment but only if the cough lasts more than 4 weeks, which was consistent with existing guidelines. ${ }^{15} \mathrm{CRS}$ took somewhat different approach and linked the cough with disturbance of Qi with exogenous pathogenic factors, which suggested to correct with Chinese medicine and acupuncture. It has to be noted, that dietary recommendations were included in management of coughing illness.

It is obvious that main concepts of management of cough suggested by TCM are dramatically different from our accepted ways and may sound somewhat unusual and difficult to understand. Let us also admit that western pediatricians, for the most part are unfamiliar with TCM concepts. In the view of it, simple rejection of TCM principles without attempt to investigate their validity, in our opinion, is unwise. There have been occurrences when initially skeptical attitude towards TCM had to be changed to more welcoming after vigorous scientific investigation. Recently published in PLoS One review of English and Chinese databases showed that Andrographis Paniculata, which has been traditionally used in Chinese herbal medicine for improvement of cold symptoms was beneficial and safe in reducing acute respiratory tract infection symptoms, and specifically sore throat and cough in children and adults. ${ }^{16}$ It may suggest that carefully selected "cough and cold" medications could be considered for treatment of acute viral cough in children.

It has to be noted that attitude of western pediatricians towards integrative medicine has not been overtly negative at all. In fact, the survey conducted by American Academy of Pediatrics (AAP) among AAP Fellows showed that $54 \%$ of participants felt that pediatricians should consider the use of all potential therapies, not just those of mainstream medicine, when treating patients. ${ }^{17}$

In conclusion, TCM may provide important and useful insight on treatment of pediatric respiratory diseases. Collaboration between western and TCM pediatricians may be beneficial for medical practice and science and should be encouraged.

\section{References}

1. JM Marchant, PA Newcombe, EF Juniper, JK Sheffield, SL Stathis, AB Chang. What is the burden of chronic cough for families? Chest 2008;134(2): 303-09.

2. AH Morice. Epidemiology of cough. Pulmonary pharmacology \& therapeutics 2002;15(3): 253-9.

3. AB Chang, JJ Oppenheimer, M Weinberger, CC Grant, BK Rubin, RS Irwin. Etiologies of Chronic Cough in Pediatric Cohorts: CHEST Guideline and Expert Panel Report. Chest 2017;152(3): 607-17.

4. AB Chang, JJ Oppenheimer, RS Irwin. Managing Chronic Cough as a Symptom in Children and Management Algorithms: CHEST Guideline and Expert Panel Report. Chest 2020.

5. MD Shields, A Bush, ML Everard, S McKenzie, R Primhak. BTS guidelines: Recommendations for the assessment and management of cough in children. Thorax 2008: iii1-iii15.

6. AB Chang, WB Glomb. Guidelines for evaluating chronic cough in pediatrics: ACCP evidence-based clinical practice guidelines. Chest 2006;129: 260S-83S.

7. DB Horton, T Gerhard, BL Strom. Trends in Cough and Cold Medicine Recommendations for Children in the United States, 2002-2015. JAMA pediatrics 2019. 
8. H Jiang, B Mao, L Wang, et al. The efficacy of QingfengGanke granule in treating postinfectious cough in pathogenic wind invading lungs syndrome: a multicenter, randomized, double-blind, placebo-controlled trial. Chinese medicine 2015;10: 21.

9. YH Yeh, YJ Chou, N Huang, C Pu, P Chou. Seasonal variations of prescriptions for the major syndrome types and manifestations of upper respiratory tract infection in tradition Chinese medicine. Complementary therapies in medicine 2016;29: 213-18.

10. W Liu, HL Jiang, B Mao. Chinese herbal medicine for postinfectious cough: a systematic review of randomized controlled trials. Evidence-based complementary and alternative medicine : eCAM 2013;2013: 906765 .

11. A Wirz-Ridolfi. The History of Ear Acupuncture and Ear Cartography: Why Precise Mapping of Auricular Points Is Important. Medical acupuncture 2019;31(3): 145-56.

12. Wirz-Ridolfi A. Resolving Unsuccessful Ear Acupuncture by Treating Focus Obstacles: A Review of the Advantages of European Ear Acupuncture. Medical Acupuncture 2014;26(3): 173-79.

13. Strittmater B. Identifying and Treating Blockages to Healing, New Approaches to Therapy-Resistant Patients. New York: Thieme 2003.

14. GS Huang, MY Hong. Genomic expression for rat model of damp obstruction in Chinese medicine: application of microarray technology. The American journal of Chinese medicine 2005;33(3): 459-74.

15. AB Chang, JJ Oppenheimer, MM Weinberger, et al. Management of Children With Chronic Wet Cough and Protracted Bacterial Bronchitis: CHEST Guideline and Expert Panel Report. Chest 2017;151(4): 884-90.

16. XY Hu, RH Wu, M Logue, et al. Andrographis paniculata (Chuān Xīn Lián) for symptomatic relief of acute respiratory tract infections in adults and children: A systematic review and meta-analysis. PloS one 2017;12(8): e0181780.

17. H McClafferty, S Vohra, M Bailey, et al. Pediatric Integrative Medicine. Pediatrics 2017;140(3). 